\title{
Stealth-nanoparticle strategy for enhancing the efficacy of steroids in mice with noise-induced hearing loss.
}

\section{$\operatorname{AUTHOR}(\mathrm{S})$ :}

Horie, Rie T; Sakamoto, Tatsunori; Nakagawa, Takayuki; Ishihara, Tsutomu; Higaki, Megumu; Ito, Juichi

\section{CITATION:}

Horie, Rie T ... [et al]. Stealth-nanoparticle strategy for enhancing the efficacy of steroids in mice with noise-induced hearing loss.. Nanomedicine 2010, 5(9): 1331-1340

\section{ISSUE DATE:}

2010-11

URL:

http://hdl.handle.net/2433/156290

\section{RIGHT:}

(c) 2010 Future Medicine Ltd.; この論文は出版社版でありません。引用 の際には出版社版をご確認ご利用ください。; This is not the published version. Please cite only the published version. 
Stealth-nanoparticle strategy for enhancing the efficacy of steroids in mice with

$$
\text { noise-induced hearing loss }
$$

Rie T Horie M.D.1, Tatsunori Sakamoto M.D., Ph.D. 1, Takayuki Nakagawa M.D., Ph.D1., Tsutomu Ishihara Ph.D.2, Megumu Higaki M.D., Ph.D.3, Juichi Ito M.D., Ph.D. 1

1 Department of Otolaryngology, Head and Neck Surgery, Graduate School of Medicine, Kyoto University, Kyoto, Japan

2 Research Institute for Drug Discovery, Graduate School of Medical Sciences, Kumamoto University, Kumamoto, Japan

3 Institute of Drug Delivery System, Research Center for Medical Sciences, Jikei University School of Medicine, Tokyo, Japan

Corresponding author:

Takayuki Nakagawa M.D., Ph.D

Department of Otolaryngology, Head and Neck Surgery

Graduate School of Medicine, Kyoto University,

Kawaharacho 54, Shogoin, Sakyo-ku, Kyoto 606-8507,Kyoto, Japan

Tel: +81-75-751-3346; Fax: +81-75-751-7225

E-mail: tnakagawa@ent.kuhp.kyoto-u.ac.jp 


\section{Abstract}

Aims: This study aimed to investigate the efficacy of encapsulating steroids, which is a primary choice for the treatment of sensorineural hearing loss (SNHL), in polyethylene glycol (PEG)-coated poly lactic acid (PLA) nanoparticles for drug delivery to the cochlea. Materials \& Methods: We prepared PEG-coated PLA nanoparticles encapsulating rhodamine or betamethasone phosphate (BP), and administered them systemically to $\mathrm{CBA} / \mathrm{N}$ mice previously exposed to intense noise. We assessed nanoparticle distribution using rhodamine fluorescence, BP concentrations in tissues, nuclear translocation of glucocorticoid receptors and the function and histology of the mouse cochleae. Results and Conclusions: PEG-coated PLA nanoparticles delivered BP to cochleae over a sustained period, resulting in significant reductions in histological and functional damage to cochleae and indicating the potential therapeutic benefits of these nanoparticles for enhancing the delivery of BP in acute SNHL.

Keywords: Drug delivery, Glucocorticoid, Hair cell, Cochlea, Hearing impairment, Noise trauma 


\section{Body of the Article}

\section{Introduction}

Sensorineural hearing loss (SNHL) is one of the commonest disabilities, for which at present we have limited therapeutic options. Many investigations have examined novel therapeutic strategies for SNHL, and have identified several agents with therapeutic activity in experimental SNHL. However, for chronic or slowly progressive SNHL, therapeutic options are limited to hearing aids and cochlear implants. Acute or progressive SNHL has a considerable impact upon an individual's quality of life. For acute SNHL, the use of systemic steroids has been a primary therapeutic choice; however, no significant hearing recovery is achieved in approximately half of the patients treated $[1,2]$. In addition, there is no evidence that explains the mechanism for the efficacy of systemic steroids in acute SNHL $[3,4,5]$. Recently, several experimental studies have demonstrated the presence of glucocorticoid receptors in the cochlea $[6,7]$, and some clinical trials have shown the efficacy of local steroid application in treating acute SNHL $[8,9]$. These results showed that therapeutic targets for steroids are present in the cochlea, suggesting the potential importance of targeted, sustained delivery of steroids in the ear.

Recent advances in drug delivery systems have produced several techniques that can be applied to the treatment of the inner ear. Encapsulating bioactive molecules in nanoparticles made from biodegradable polymers such as polylactic acid (PLA) and polylactic/glycolic acid (PLGA) allows the sustained release of bioactive molecules in a controlled manner [10]. Previously, we examined the potential of systemically applied PLGA/PLA nanoparticles for delivering drugs to the cochlea, but found that these nanoparticles did not significantly target the drugs, and that most of the PLGA/PLA nanoparticles were metabolized in the liver [11]. Recently, stealth-nanoparticles, in which PLA is coated with poly-ethylene glycol (PEG), have been developed. PEG-coated PLA 
nanoparticles overcome the problems associated with conventional nanoparticles by reducing opsonization and preventing interactions with the reticuloendothelial system in the liver or spleen [12-14]. This study aimed to investigate the efficacy of PEG-coated PLA nanoparticles, stealth nanoparticles, for delivering steroids to the cochlea and their therapeutic potential for the treatment of noise-induced hearing loss. We systemically administered PEG-coated PLA nanoparticles containing betamethasone phosphate (BP) or the red fluorescent dye, Rhodamine B, to mice that had been exposed to intense noise, and examined the distribution of rhodamine fluorescence, the BP concentrations in tissues, and the function and histology of the mouse cochleae.

\section{Materials \& Methods}

Experimental animals

Male CBA/N mice, 4-6 weeks old and weighing 18 to $22 \mathrm{~g}$, were purchased from Japan SLC Inc. (Hamamatsu, Japan). The Animal Research Committee at the Graduate School of Medicine, Kyoto University approved all experimental protocols, and animal care was supervised by the Institute of Laboratory Animals at the Graduate School of Medicine, Kyoto University. All experimental procedures were performed in accordance with National Institute of Health $(\mathrm{NIH})$ guidelines for the care and use of laboratory animals.

\section{PEG-coated PLA nanoparticles}

Poly D, L-lactic acid (PLA) was purchased from Wako Pure Chemicals Industries, Ltd. (Osaka, Japan). Polyethylene glycol (PEG)-block-PLA was synthesized by a ring-opening polymerization of D, L-lactide, which had been purified by re-crystallization in ethyl acetate, in the presence of monomethoxy-PEG (MW 5,580, NOF Co., Tokyo, Japan). 
PEG-coated PLA nanoparticles were prepared by the oil-in-water solvent diffusion method as described elsewhere $[14,15]$. In brief, a mixture of $7.8 \mathrm{mg}$ PEG-PLA and $42.2 \mathrm{mg}$ PLA was dissolved in $1 \mathrm{ml}$ of acetone. To this solution, $500 \mu \mathrm{l}$ of an acetone solution of DEA $(15 \mathrm{mg} / \mathrm{ml})$, followed by $68 \mu \mathrm{l}$ of an aqueous solution of zinc chloride (1 M; pH 1.9), and then $28 \mu \mathrm{l}$ of an aqueous solution of BP $(350 \mathrm{mg} / \mathrm{ml})$ or $50 \mu \mathrm{l}$ ethanol containing Rhodamine B $(20 \mathrm{mg} / \mathrm{ml}$, Sigma-Aldrich) were added; the mixture was then allowed to stand for $30 \mathrm{~min}$ at room temperature.) A 26-gauge needle was used to add the mixture drop-wise to distilled water stirred at $1000 \mathrm{rpm}$, and $0.5 \mathrm{M}$ citrate (Wako Pure Chemicals) and Tween 80 (Wako Pure Chemicals) were added immediately to chelate BP-zinc complexes. Finally, the PEG-coated PLA nanoparticles were purified and concentrated by ultrafiltration (Centriprep YM-50; Millipore Corporation, Bedford, MA). We prepared PEG-coated PLA nanoparticles encapsulating Rhodamine B (Stealth-Nano-Rhodamine) with an average diameter of $120 \mathrm{~nm}$ and a loading efficiency of rhodamine in the nanoparticles of $0.1 \%$ (wt/wt) and nanoparticles encapsulating BP (Stealth-Nano-BP) with an average diameter of $116 \mathrm{~nm}$ and a loading efficiency of BP in the nanoparticles of $10.7 \%(\mathrm{wt} / \mathrm{wt})$.

\section{Noise exposure and drug administration}

Baseline auditory brain stem response (ABR) thresholds were measured within $7 \mathrm{~d}$ of the mice being exposed to the initial noise, which was $8 \mathrm{KHz}$ octave band noise at a sound pressure level (SPL) of $120 \mathrm{~dB}$ for $2 \mathrm{~h}$. This was done in a ventilated sound exposure chamber with the mice under general anesthesia, using $10 \mathrm{mg} / \mathrm{kg}$ midazolam, $37.5 \mathrm{mg} / \mathrm{kg}$ medetominide, and $0.5 \mathrm{mg} / \mathrm{kg}$ butorphanol tartrate. Sound levels were monitored and calibrated at multiple locations within the sound chamber to ensure stimulus uniformity.

After exposure to traumatic noise, the animals received intravenous injections into the tail vein 
of $200 \mu \mathrm{l}$ of a $3 \mu \mathrm{g} / \mathrm{ml}$ solution of Rhodamine B $(\mathrm{n}=6), 200 \mu \mathrm{l}$ Stealth-Nano-Rhodamine solution, containing the same total mass of Rhodamine B as administered to the mice given Rhodamine $\mathrm{B}$ alone $(\mathrm{n}=6), 200 \mu 1$ of a $250 \mu \mathrm{g} / \mathrm{ml}$ BP solution $(\mathrm{n}=12), 200 \mu 1$ Stealth-Nano-BP solution, containing the same total mass of BP as administered to the mice given BP alone ( $\mathrm{n}=12)$, or physiological saline $(n=4)$.

\section{Rhodamine distribution}

The animals that had been treated with Rhodamine B or Stealth-Nano-Rhodamine were sacrificed 2 or $24 \mathrm{~h}$ after drug administration by asphyxiation in $\mathrm{CO}_{2}$ for $15 \mathrm{~min}$. Cochleae were harvested from the mice ( $\mathrm{n}=4$ for each group), immersed in $4 \%$ paraformaldehyde in phosphate-buffered saline (PBS) for $4 \mathrm{~h}$, and then decalcified in $0.1 \mathrm{M}$ ethylenediaminetetraacetic acid (EDTA) in PBS for $4 \mathrm{~d}$ at $4^{\circ} \mathrm{C}$. The liver was dissected from each animal and processed in the same way as cochlear specimens. Tissue specimens were cut into $10 \mu \mathrm{m}$ thick sections. Two mid-modiolus sections from the cochlea of each animal and two randomly selected sections from the liver were used for histological analysis. Nuclei were stained with 4',6-diamidino-2-phenylindole dihydrochloride (DAPI, Invitrogen, Carlsbad, CA). The specimens were mounted in Vector Shield (Vector Laboratories Inc., Burlingame, CA), and viewed with a fluorescence microscope (BX50, Olympus, Tokyo, Japan).

\section{Betamethasone concentration}

Animals that had been given BP or Stealth-Nano-BP were assessed for BP concentrations in the cochlea and liver. The cochleae and livers were collected under general anesthesia 1, 12 or $24 \mathrm{~h}$ after drug administration ( $\mathrm{n}=4$ for each time point). Immediately after dissection, specimens were homogenized in PBS, and then stored at $-80{ }^{\circ} \mathrm{C}$. The tissue concentration of BP was quantified using 
a time-resolved fluoroimmunoassay (TR-FIA) kit for betamethasone (Shionogi \& Co., Ltd., Osaka, Japan) as described elsewhere [10]. The detection limit for BP in this assay was $0.01 \mu \mathrm{g} / \mathrm{ml}$.

\section{Immunohistochemistry for glucocorticoid receptor}

Animals received an intravenous injection of Saline, BP or Stealth-Nano-BP immediately after noise exposure ( $\mathrm{n}=4$ each condition). Cochleae were harvested from the mice $24 \mathrm{~h}$ after noise exposure, fixed with $4 \%$ PFA for $1 \mathrm{~h}$ and prepared as frozen sections in $10 \mu \mathrm{m}$ thickness. Three mid-modiolar sections separated by $30 \mu \mathrm{m}$ from each cochlea were provided for immunohistochemistry for glucocorticoid receptor (GR). A polyclonal rabbit anti-GR antibody $(5 \mathrm{mg} / \mathrm{ml}$; Affinity Bioreagents, Carlsbad, CA) was used as the primary antibody, and Alexa-594-conjugated anti-rabbit goat IgG (1:500; Invitrogen, Carlsbad, CA) was used as the secondary antibody. Nuclear staining with DAPI was performed. For quantitative analyses To quantify the nuclear translocation of GR from the cytoplasm in hair cells, the numbers of inner hair cells (IHCs) and outer hair cells (OHCs) with immunoreactivity for GR in the nuclei were counted using the mid-basal portion of cochleae, respectively. We defined GR translocation rate (\%) as (The number of GR translocated hair cells) / (The number of counted hair cells) x $100 \%$. The average value in three sections from each cochlea was used the data for the animal.

\section{Auditory function}

Auditory function was assessed using ABR recordings. ABR thresholds were measured at frequencies of 8,16 and $32 \mathrm{kHz} 5 \mathrm{~min}$ after drug administration and again after 4, 7 and 14 days. Animals that had been given BP $(n=4)$, Stealth-Nano-BP $(n=4)$ or saline $(n=4)$ were anesthetized using midazolam, medetominide and butorphanol tartrate, and kept warm with a heating pad. Acoustic stimuli were generated and the evoked potentials were recorded using a PowerLab/4sp (AD 
Instruments, Castle Hill, Australia). Acoustic stimuli, consisting of tone-burst stimuli (for $0.1 \mathrm{~ms}$ with a $\cos 2$ rise/fall and $1 \mathrm{~ms}$ plateau), were delivered to one ear though a speaker (ES1spc; Bioresearch Center, Nagoya, Japan) connected to a funnel fitted into the external auditory meatus. To record bioelectrical potentials, stainless steel needle electrodes were inserted subdermally at the vertex (ground), and ventrolateral (active) and contralateral (reference) to the ear being monitored. Stimuli were calibrated against a 1/4-inch free-field microphone (ACO-7016; ACO Pacific, Belmont, CA) connected to an oscilloscope (DS-8812 DS-538; Iwatsu Electric, Tokyo, Japan) or a sound level meter (LA-5111; Ono Sokki, Yokohama, Japan). Responses between the vertex and mastoid subdermal electrodes were amplified using a digital amplifier (MA2; Tucker-Davis Technologies, Alachua, FL). Thresholds were determined from a set of responses at varying intensities at $5 \mathrm{~dB}$ SPL intervals and electrical signals were averaged over 1024 repetitions. Threshold measurements at each frequency were repeated at least twice.

\section{Hair cell protection}

After ABR measurements had been made 14 days after drug administration, the animals were anesthetized with lethal dose of anesthetics and given intracochlear perfusions of $4 \%$ paraformaldehyde in PBS. The temporal bones were excised and decalcified by immersion in the same fixative at $4^{\circ} \mathrm{C}$ for $4 \mathrm{~h}$, and then in $0.1 \mathrm{M}$ EDTA in PBS. Hair cell damage was evaluated in whole mounts from groups of mice $(n=4)$ treated with BP, Stealth-Nano-BP, and saline. F-actin was stained with $3 \mu \mathrm{g} / \mathrm{ml}$ fluorescein isothiocyanate (FITC)-conjugated phalloidin (Sigma-Aldrich) to identify cochlear hair cells in the whole mounts. These whole mounts were inspected using a Leica TCS SPE confocal microscope (Leica Microsystems Inc., Wetzlar, Germany) and the number of remaining IHCs and OHCs were counted. We defined apical as the region $20-40 \%$ from the apex and basal as the region $60-80 \%$ from the apex. The numbers of IHCs and OHCs were counted in a 
$0.2 \mathrm{~mm}$ long strip in the apical and the basal portions of each cochlea.

\section{Statistical Analyses}

All statistical analyses used GraphPad Prisms ${ }^{\circledR}$ (GraphPad Software, La Jolla, CA). The statistical assessment of BP concentrations and ABR thresholds used two-way analysis of variance (ANOVA) followed by multiple t-tests with Bonferroni corrections. The numbers of IHCs and OHCs, and GR translocation rates were assessed using one-way ANOVA followed by Tukey's multiple comparison tests. P values below 0.05 were considered significant. Values were expressed as the means \pm standard deviation.

\section{Results}

Rhodamine distribution

After administering free Rhodamine B to mice, a few red dots of rhodamine fluorescence were observed in the liver after $15 \mathrm{~min}$, numerous red fluorescence dots were found after $2 \mathrm{~h}$, and no fluorescence was visible after $24 \mathrm{~h}$ (Fig. 1A-C). In contrast, in mice treated with Stealth-Nano-Rhodamine, red fluorescence dots were visible in the liver $15 \mathrm{~min}, 2$ and $24 \mathrm{~h}$ after administration (Fig. 1A'-C'), with obvious accumulation of rhodamine fluorescence in the liver at 24 h. These results indicated a delay in the metabolism of Rhodamine B encapsulated in PEG-coated PLA nanoparticles by the reticuloendothelial system in the liver.

In the cochlea, no rhodamine fluorescence was observed after the administration of free Rhodamine B (Fig. 1 D-F)), as we had previously observed in normal guinea pigs [11]. In contrast, red fluorescence dots were observed in the cochleae of mice given Stealth-Nano-Rhodamine at 15 min, $2 \mathrm{~h}$ and $24 \mathrm{~h}$ after treatment (Fig. 1D'-F'). Rhodamine fluorescence was located in the stria vascularis and spiral prominence in all the cochlear turns, and in the cochlear modiolus, which 
correspond to the locations of blood vessels in the cochlea. These results suggested that encapsulating drugs in PEG-coated PLA nanoparticles could be an effective method for delivering drugs to the cochlea.

\section{Betamethasone concentrations}

We assessed betamethasone concentrations in the liver and cochlea at 1, 12 and $24 \mathrm{~h}$ after BP or Stealth-Nano-BP had been systemically administered to mice. TR-FIA analyses revealed significantly higher BP concentrations in the livers of mice given Stealth-Nano-BP than those given free BP, at each time point (Fig. 2A). In addition, the BP concentrations did not significantly drop between 1 and 12 or $24 \mathrm{~h}$ in mice given Stealth-Nano-BP, while they did in mice given free BP (Fig. 2A), supporting the hypothesis that encapsulating BP in PEG-coated PLA nanoparticles effectively enables the drug to escape metabolism by the reticuloendothelial system in the liver.

In the cochlea, BP was detectable by the TR-FIA method in all the experimental groups. In the cochleae of mice treated with free BP, the concentrations were low $(4.44 \pm 1.69 \mathrm{ng} /$ cochlea $)$ even $1 \mathrm{~h}$ after BP application and dropped to extremely low concentrations after $12 \mathrm{~h}(0.07 \pm 0.03$ $\mathrm{ng} /$ cochlea $)$ and $24 \mathrm{~h}(0.17 \pm 0.14 \mathrm{ng} /$ cochlea $)$. In contrast, the BP concentrations measured in the cochleae of mice treated with Stealth-Nano-BP were much higher. The BP concentrations were $21.57 \pm 2.55 \mathrm{ng} /$ cochlea after $1 \mathrm{~h}, 10.45 \pm 3.92$ after $12 \mathrm{~h}$ and $9.69 \pm 3.65$ after $24 \mathrm{~h}$, which were significantly higher than in mice treated with free BP at every time point (Fig. 2B). In contrast to the liver, in the cochlea the BP concentration $12 \mathrm{~h}$ after Stealth-Nano-BP administration was significantly lower than $1 \mathrm{~h}$ after administration (Fig. 2B). However, comparatively high BP concentrations were maintained between 12 and $24 \mathrm{~h}$ in these mice, with no significant difference between the BP concentrations at 12 and $24 \mathrm{~h}$ after treatment (Fig. 2B). Even $24 \mathrm{~h}$ after Stealth-Nano-BP treatment, the BP concentration in the cochlea was significantly higher than at $1 \mathrm{~h}$ 
after free BP treatment. These results support the hypothesis that encapsulating BP in PEG-coated PLA nanoparticles is an effective strategy for sustained BP delivery to the cochlea.

\section{GR translocation in hair cells}

We evaluated nuclear translocation of GRs after acoustic trauma to determine whether BP that had been systemically administered actually activates GR in hair cells or not. Immunostainig for GR demonstrated GR distribution in hair cells of cochleae $24 \mathrm{~h}$ after noise exposure. The majority of hair cells that were treated with Stealth-Nano-BP exhibited GR immunoreactivity in the nucleus and cytoplasm (Fig. 3A, A'), while in saline-treated cochleae, most of hair cells showed GR immunostaining only in the cytoplasm (Fig. 3B, B'). GR translocation rates in OHCs of Stealth-Nano-BP- and BP-treated cochleae were $72.22 \pm 6.42$ and $19.44 \pm 16.67 \%$. No OHCs of saline-treated cochleae showed GR translocation into the nuclei. Differences in GR translocation rate in OHCs between Stealth-Nano-BP- and BP-treated cochleae, and Stealth-Nano-BP- and saline-treated cochleae were statistically significant (Fig. 3C). GR translocation rates in IHCs of Stealth-Nano-BP-, BP- and saline-treated cochleae were $83.33 \pm 33.33,66.67 \pm 47.14$ and 41.67 $\pm 50.00 \%$. No significant differences in GR translocation rates in IHCs among experimental groups were found (Fig. 3C). These findings indicate that BP delivered from Stealth-Nano-BP actually activates GRs in hair cells.

\section{Auditory function}

We used conventional ABR recordings to monitor auditory function after exposure to traumatic noise. Time courses of the changes in ABR thresholds after drug treatment, at 8,16 and $32 \mathrm{KHz}$, are shown in Fig. 3. Drug treatment showed significant effects on the ABR thresholds at each frequency with two-way ANOVA. Multiple comparison analyses using the Bonferroni test identified specific, 
significant differences between the groups of mice given Stealth-Nano-BP or BP on day 14 after treatment at $8 \mathrm{KHz}$, and on days 7 and 14 after treatment at $32 \mathrm{KHz}$; specific significant differences were also identified between the groups of mice treated with Stealth-Nano-BP or saline on days 4 and 14 after treatment at $8 \mathrm{KHz}$. In contrast to Stealth-Nano-BP, BP treatment showed no significant effects on the attenuation of ABR thresholds compared to saline injection (Fig. 4). These results showed that Stealth-Nano-BP had better therapeutic effects on noise-induced hearing impairment than free BP.

\section{Hair cell protection}

We examined the cochlear sensory epithelia histologically and counted the surviving hair cells, to investigate the ability of Stealth-Nano-BP to protect these cells at a histological level. Phalloidin staining showed well-ordered rows of hair cells, three rows of OHCs and a single row of IHCs, in cochlear epithelia from mice treated with Stealth-Nano-BP (Fig. 5A), while a loss of both IHCs and OHCs was notable in both the apical and basal portions of cochleae from mice treated with BP or saline (Fig. 5B, C). Quantitative assessments using one-way ANOVA showed that drug treatment had significant effects on the numbers of IHCs in the apical and basal regions and on the number of OHCs in the apical region, but not in the basal region of the cochlea (Fig. 5D, E). Tukey's multiple comparison tests showed significant differences in the number of IHCs in the apical region between mice treated with Stealth-Nano-BP and BP or saline and in the number of IHCs in the basal region between mice treated with Stealth-Nano-BP and saline (Fig. 5D). There was also a significant difference in the number of OHCs in the apical region between mice treated with Stealth-Nano-BP and saline (Fig. 5E). These results showed that Stealth-Nano-BP had a greater potential as a therapeutic to protect hair cells against noise-induced damage than free BP. 


\section{Discussion}

BP is one of the most widely used steroids in the clinic for the treatment of acute SNHL, including noise-induced SNHL. In this study, we have shown that a single injection of BP at a dose of approximately $2.5 \mathrm{mg} / \mathrm{kg}$, which is clearly a much higher dose than is generally used in the clinic, had no significant effect on the function and the histology of mouse cochleae damaged by noise. Strikingly, the intra-cochlear concentration of BP after a free BP injection was incredibly low, even $1 \mathrm{~h}$ after the administration of such a high dose, and despite the serum concentration of BP reaching a peak $1 \mathrm{~h}$ after systemic administration [16]. This suggested that single injections of BP at the doses used clinically may deliver very limited amounts of BP into the cochlea, and this may account for the poor effects of glucocorticoids seen in patients with acute SNHL. It is certainly consistent with the lack of effect on noise-induced trauma to the cochlea seen in this study after a single intravenous injection of BP. Previously, protective effects of glucocorticoids on noise-induced hearing loss have widely been investigated. However, the results vary depending on used administration routes (systemic or local) and doses, intensities of exposed noise or species of experimental animals. Among previous studies, two reports have been published using similar experimental setting of the present study, in which mice are used experimental animals and glucocorticoid is systemically administered $[17,18]$. These two studies show significant protection of auditory function by systemic application of glucocorticoids; however, the dose of glucocorticoids applied or the severity of noise trauma in these studies are quite different from that used in the present study. We therefore consider that such differences may vary outcomes in hearing protection by glucocorticoids.

Nanoparticle technology has been included in potential strategies for improving delivery of therapeutic agents including glucocorticoids to the cochlea. We previously studied the efficacy of a non-stealth type of nanoparticles for cochlear drug delivery, and the results indicated limited 
capacity of non-stealth nanoparticles for sustained and/or targeting delivery of drugs to cochleae after systemic application [11], which may be due to rapid removal of non-stealth nanoparticles from circulation by the mononuclear phagocyte system (MPS) in the liver and spleen. PEG is an attractive material for surface modification of PLA or PLA/PLGA nanoparticles to reduce interactions with the MPS system. PLA or PLA/PLGA nanoparticles with PEG coating efficiently escape from the MPS resulting in prolonged circulation of PLA or PLA/PLGA nanoparticles [12-14]. Recent progress in this field enables preparing a variety of PEG-coated PLA or PLA/PLGA nanoparticles with different diameters, blend ratios and molecular weights [19]. Stealth-Nano-BP used in the present study releases a half of loaded BPs for 36 days in vitro and their T1/2 is $7.8 \mathrm{~h}$ after intravenous injection [19]. In the present study, a single injection of Stealth-Nano-BP resulted in high BP concentrations in the cochlea, which at $1 \mathrm{~h}$ after treatment were four-fold higher than for free BP. In addition, $12 \mathrm{~h}$ after the administration of Stealth-Nano-BP, the BP concentration in the cochlea remained at half of that seen after $1 \mathrm{~h}$, whereas virtually no BP was detectable $12 \mathrm{~h}$ after injecting free BP. Moreover, even $24 \mathrm{~h}$ after the administration of Stealth-Nano-BP, the BP concentration seen after $12 \mathrm{~h}$ was sustained. These results have shown that Stealth-Nano-BP is effective for the sustained delivery of high concentrations of BP to the cochlea. In addition, immunohistochemistry for GR in the present study demonstrates higher incidence of the nuclear translocation of GRs in hair cells following Stealth-Nano-BP application than free BP application, which indicates that BP released from Stealth-Nano-BP actually activates GRs in hair cells and that this results in functional and histological protection of the cochlea from the trauma resulting from loud noise.

Two possible mechanisms are aroused for high concentrations of BP in cochleae following administration of Stealth-Nano-BP. One is an increase of blood circulation time of BP-loaded nanoparticles by the stealth effect, escaping of Stealth-Nano-BP from the MPS resulting in an increase of blood circulation time of BP. Another is the accumulation of Stealth-Nano-BP in the 
cochlear capillaries. Our previous study using non-stealth nanoparticles encapsulating rhodamine showed limited deliver of non-stealth nanoparticles into the cochlear capillaries even 10 min after application, with virtually no rhodamine fluorescence identified in cochleae $2 \mathrm{~h}$ after application [11]. In contrast to non-stealth nanoparticles, histological findings using PEG-coated PLA nanoparticles loaded rhodamine demonstrate the presence of PEG-coated PLA nanoparticles in the cochlear capillaries even $24 \mathrm{~h}$ after systemic application. We therefore consider that polymeric nanoparticles are capable to accumulate in the cochlear capillaries under pathological conditions, if sufficient amounts of nanoparticles are supplied in circulation. In addition, noise trauma is known to induce a decrease of the cochlear blood flow velocity [20], which can be a possible mechanism for prolonged presence of PEG-coated PLA nanoparticles. Some previous studies have indicated that noise trauma damages a blood-labyrinth barrier resulting in an increase of drug entry into cochlear tissues $[21,22]$. Contradictorily, another study demonstrates no significant change in the blood-labyrinth barrier after noise trauma [23]. In present findings, no rhodamine fluorescence was identified in cochlear specimens treated with PEG-coated PLA nanoparticles except for the locations corresponding to those of cochlear vascular spaces, suggesting that PEG-coated PLA nanoparticles used in the present study are not able to pass a blood- labyrinth barrier even after intense noise exposure.

Our results have shown that a single injection of Stealth-Nano-BP protected auditory function and cochlear sensory hair cells from noise trauma. We consider that this is likely to be due to the sustained delivery of $\mathrm{BP}$ at high concentrations to the cochlea achieved by using stealth-nanoparticle technology. In the clinical treatments of acute SNHL, daily injections of BP for five to ten days are given, but this generally results in unsatisfactory outcomes in terms of hearing recovery. Our measurements of cochlear BP concentrations in mice, presented here, show that free BP disappeared from the cochlea within $12 \mathrm{~h}$, while Stealth-Nano-BP could maintain higher BP concentrations in the cochlea for $24 \mathrm{~h}$ than were achieved after $1 \mathrm{~h}$ using free BP injections. All 
together, our results strongly suggest that the clinical application of Stealth-Nano-BP may improve hearing recovery in patients with acute SNHL, which would contribute to an improved quality of life for these patients. In the near future, we will investigate the efficacy of Stealth-Nano-BP in other acute SNHL models and the risk of adverse effects that may be associated with their use, in pre-clinical studies.

\section{Conclusions}

To examine the efficacy of stealth-nanoparticles encapsulating BP for the treatment of noise-induced SNHL, we have carried out pharmacokinetic, functional and histological analyses in a mouse model of noise-induced SNHL. Pharmacokinetic experiments showed the efficacy of stealth-nanoparticles for sustained drug delivery to the cochlea. Functional and histological analyses of noise-damaged cochleae demonstrated significant amelioration of noise-induced damage after the systemic application of stealth-nanoparticles encapsulating BP. These findings encourage us to perform pre-clinical studies with stealth-nanoparticles encapsulating BP for the treatment of acute SNHL.

\section{Acknowledgements}

This work was supported by a Grant-in-Aid for Researches on Sensory and Communicative Disorders and a Grant-in-Aid for Research on Nanotechnical Medical from the Japanese Ministry of Health, Labour and Welfare. 


\section{References}

1 Wilson WR, Byl FM, Laird N: The efficacy of steroids in the treatment of idiopathic sudden hearing loss. A double-blind clinical study. Arch Otolaryngol. 106(12), 772-776 (1980).

2 Kanzaki J, Inoue $\mathrm{Y}$, Ogawa $\mathrm{K}$ et al.: Effect of single-drug treatment on idiopathic sudden sensorineural hearing loss. Auris Nasus Larynx. 30(2), 123-127 (2003).

3 Conlin AE, Parnes LS: Treatment of sudden sensorineural hearing loss: . A Meta-analysis. Arch Otolaryngol Head and Neck Surg. 133(6), 582-586 (2007).

4 Ghosh A, Jackson R: Best evidence topic report. Steroids in sudden sensorineural hearing loss. Emerg Med J. 22(10), 732-733 (2005).

$5 \quad$ Wei BP, Mubiru S, O’Leary S: Steroids for idiopathic sudden sensorineural hearing loss. Cochrane Detabase Syst Rev. 25(1), (2006).

6 Canlon B, Meltser I, Johansson P et al.: Glucocorticoid receptors modulate auditory sensitivity to acoustic trauma. Hear Res. 226(1-2), 61-69 (2007).

7 Zuo J, Lisa M, Yao CX et al.: Glucocorticoid receptor expression in the postnatal rat cochlea. Hear Res. 87(1-2), 220-227 (1995).

8 Doyle KJ, Bauch C, Battista R et al.: Intratympanic steroid treatment: a review. Otol Neurotol. 25(6), 1034-1039 (2004).

9 Haynes DS, O’Malley M. Cohen $\mathrm{S}$ et al.: Intratympanic dexamethasone for sudden sensorineural hearing loss after failure of systemic therapy. Laryngoscope. 117(1), 3-15 (2007).

10 Ishihara $\mathrm{T}$, Izumo $\mathrm{N}$, Higaki $\mathrm{M}$ et al.: Role of zinc in formulation of PLGA/PLA nanoparticles encapsulating betamethasone phosphate and its release profile. J Control Release. 105(1-2), 68-76 (2005). 
11 Tamura $\mathrm{T}$, Kita $\mathrm{T}$, Nakagawa $\mathrm{T}$ et al.: Drug delivery to the cochlea using PLGA nanoparticles. Laryngoscope. 115(11), 2000-2005 (2005).

12 Gref R, Minamitake Y, Peracchia MT et al.: Biodegradable long-circulating polymeric nanospheres. Science. 263(5153), 1600-1603 (1994).

13 Stolnik S, Dunn SE, Garnett MC et al.: Surface modification of poly (lactic-co-glycolide) nanospheres by biodegradable poly (lactide)-poly (ethykene glycol) copolymers. Pharm Res. 11(12), 1800-1808 (1994).

14 Ishihara $\mathrm{T}$, Takahashi $\mathrm{M}$, Higaki $\mathrm{M}$ et al.: Prolonging the in vivo residence time of prostaglandin E(1) with biodegradable nanoparticles. Pharm Res. 25(7), 1686-1695 (2008).

15 Ishihara $\mathrm{T}$, Takahashi M, Higaki $\mathrm{M}$ et al.: Efficient of encapsulation of water-soluble corticosteroid in biodegradable nanoparticles. Int J Pharm. 365(1-2), 200-205 (2009).

16 Petersen MC, Nation RL, McBride WG et al.: Pharmacokinetics of betamethasone in healthy adults after intravenous administration. Eur J Clin Pharmacol. 25(5), 643-650 (1983).

17 Tabuchi K, Murashita H, Sakai S, et al.: Therapeutic time window of methylprednisolone in acoustic injury. Otol Neurotol. 27(8), 1176-1179 (2006).

18 Tahera Y, Meltser I, Johansson P, et al.: NF-kappaB mediated glucocorticoid response in the inner ear after acoustic trauma. J Neurosci Res. 83(6), 1066-1076 (2006).

19 Ishihara T, Kubota T, Choi T, et al.: Polymeric nanoparticles encapsulating betamethasone phosphate with different release profiles and stealthiness. Int J Pharm. 375(1-2), 148-154 (2009).

20 Nakashima T, Nakagawa S, Sone M, et al.: Disorders of cochlear blood flow. Brain Res Rev. 43, 17-28 (2003). 
21 Hashimoto K, Seki M, Miyasaka H, et al.: Effect of steroids on increased permeability of blood vessels of the stria vascularis after auditory ossicle vibration by a drill in otologic surgery. Ann Otol Rhinol Laryngol. 15(10),769-774 (2006).

22 Suzuki M, Yamasoba T, Ishibashi T, et al.: Effect of noise exposure on blood-labyrinth barrier in guinea pigs. Hear Res. 164, 12-18 (2002).

23 Laurell GF, Teixeira M, Duan M, et al.: Intact blood-perilymph barrier in the rat after impulse noise trauma. Acta Otolaryngol. 128(6), 608-612 (2008). 
Figures

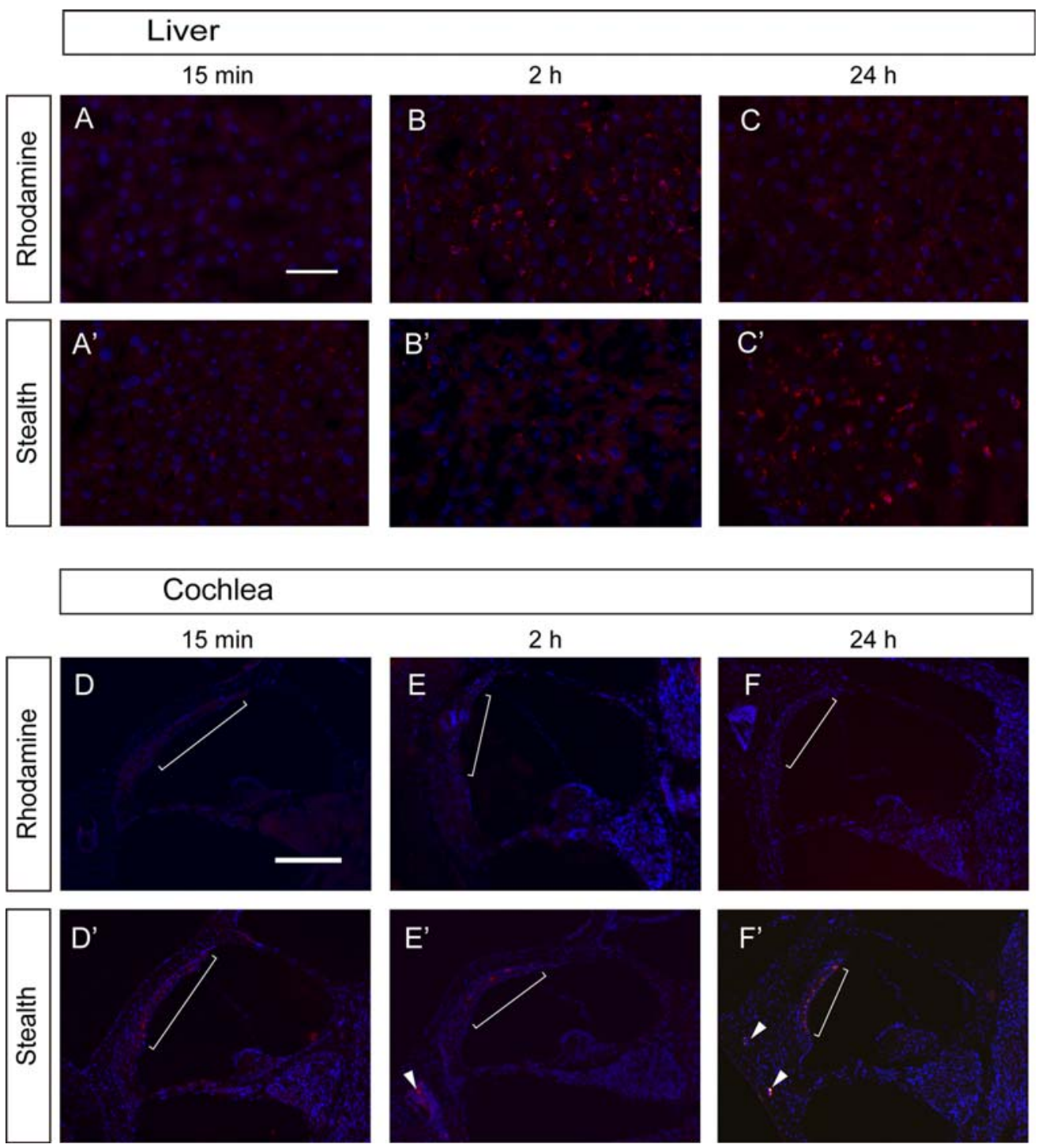

Fig.1. Rhodamine distribution in the liver and cochlea.

In the liver in mice after application of free rhodamine (A-C), numerous rhodamine fluorescence dots (red) were observed $2 \mathrm{~h}$ after application. In mice treated with Stealth-Nano-Rhodamine ( $\left.\mathrm{A}^{\prime}-\mathrm{C}^{\prime}\right)$, rhodamine fluorescence was visible at each time point. After free rhodamine application (D-F), no rhodamine fluorescence was detectable in cochleae. After application of Stealth-Nano-Rhodamine $\left(D^{\prime}-F^{\prime}\right)$, rhodamine fluorescence was seen in the stria vascularis (brackets) and in the vessels in the bony wall of the cochlea (arrow heads) at each time point. Nuclei were stained with DAPI (blue). Bars in A-C and A'-C' represent $50 \mu \mathrm{m}$ and bars in D-F and D'-F' represent $200 \mu \mathrm{m}$. 

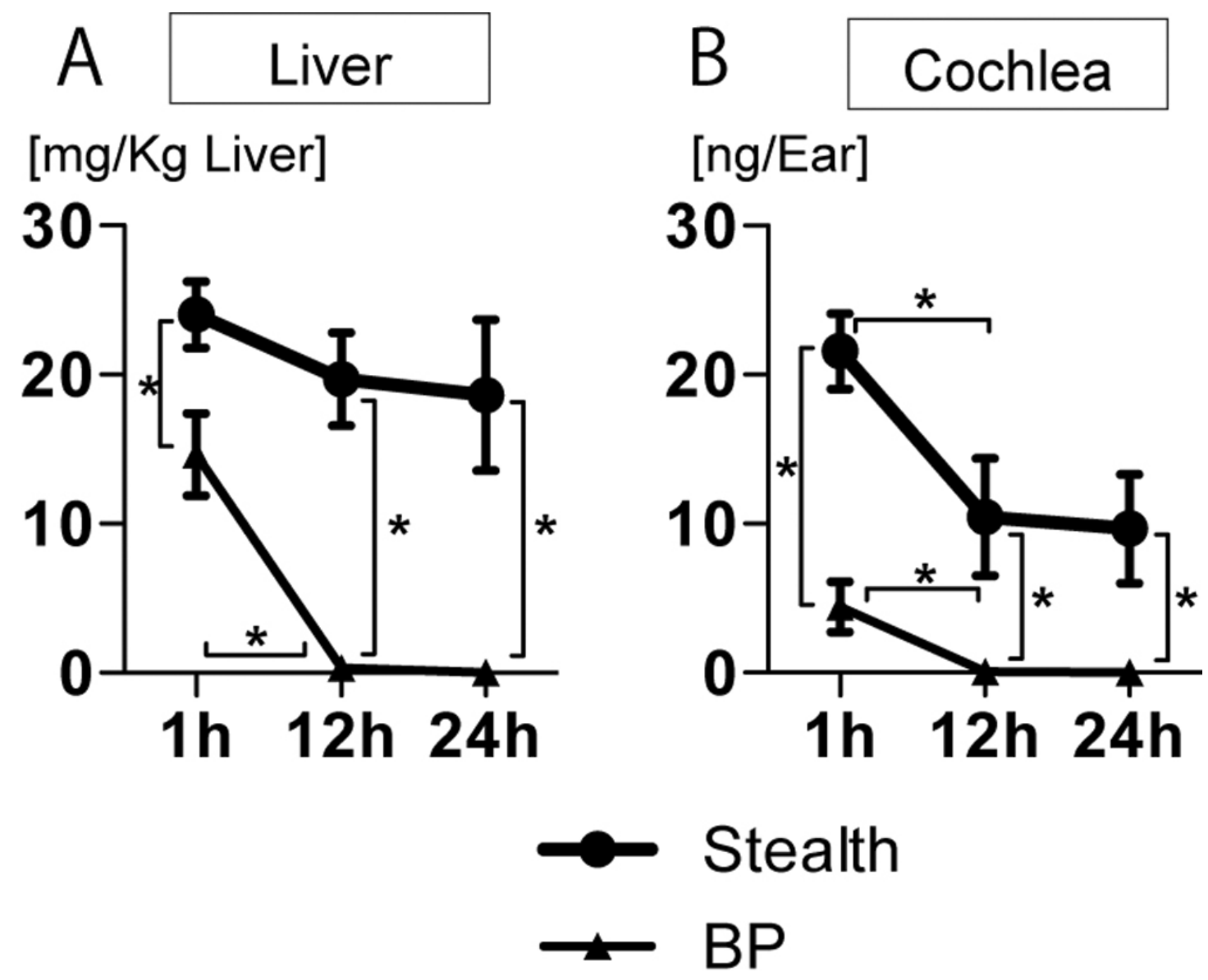

- Stealth

Fig 2. Betamethasone concentrations in the liver and cochlea

Tissue concentrations of betamethasone in the liver (A) and the cochlea (B) were determined at 1, 12 and $24 \mathrm{~h}$ after systemic application of free BP or Stealth-Nano-BP. Betamethasone concentrations after Stealth-Nano-BP application were significantly higher those after free BP application in both the liver (A) and cochlea (B) at each time point. After BP injection, betamethasone concentration significantly dropped between 1 and $12 \mathrm{~h}$. In the cochlea, samples treated with Stealth-Nano-BP also exhibited significant reduction of betamethason levels between 1 and $12 \mathrm{~h}$, but no significant reduction was not observed between 12 and $24 \mathrm{~h}(\mathrm{~B})$. Asterisks indicate statistical significance with multiple t-tests with Bonferroni corrections. Bars represent one standard deviation. 

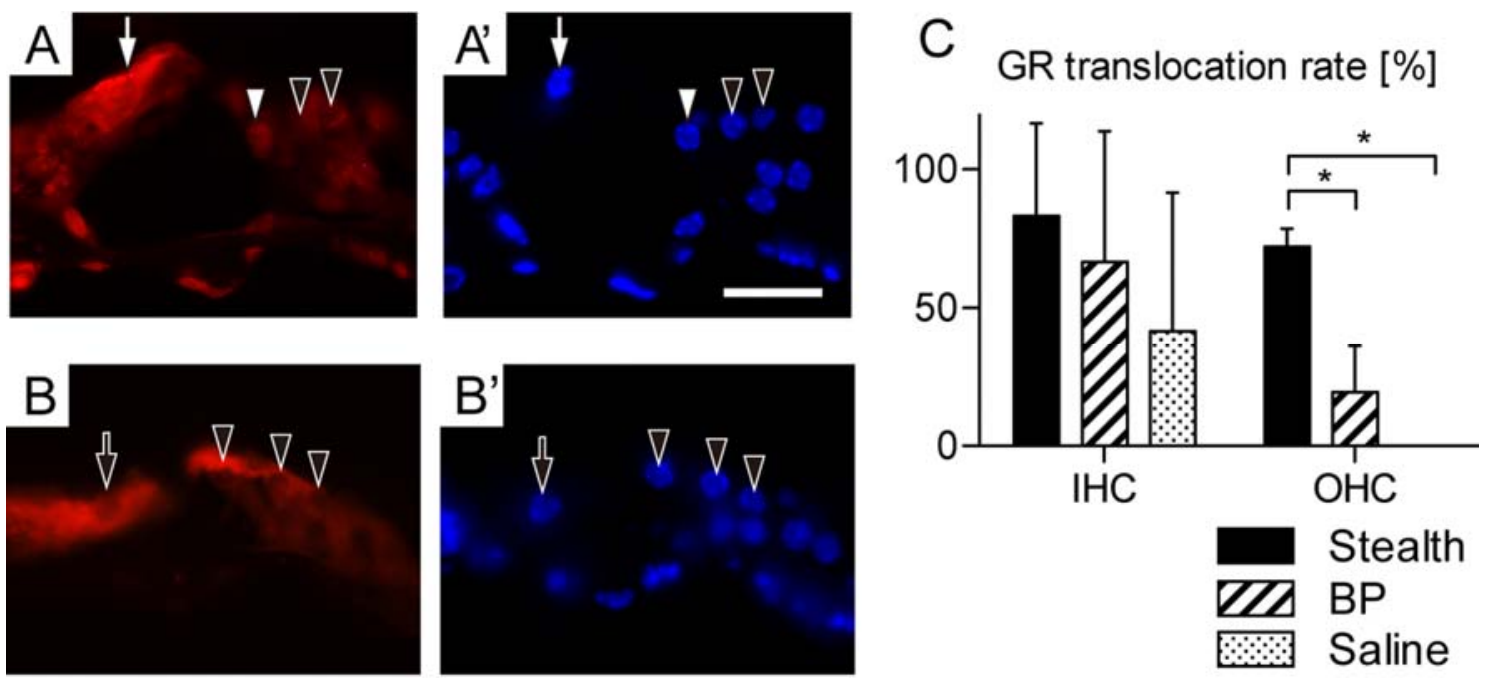

Fig. 3. Nuclear translocation of glucocorticoid receptors in cochlear hair cells.

Immunostaining for glucocorticoid receptors (GRs) demonstrates the cellular location of GRs in hair cells $(\mathrm{A}, \mathrm{B})$ and the nuclear locations are demonstrated by DAPI staining (A', B'). Arrows indicate IHCs and arrowheads indicate OHCs (A, B). White arrows or arrowheads show IHCs or OHCs with GR translocation into the nuclei, and black arrows or arrowheads show non-translocated IHCs or OHCs (A, B). Bar in (A') represents $20 \mu \mathrm{m}$ for A-B'. GR translocation rates in IHC and OHC for cochleae treated with Stealth-Nano-BP (Stealth), free BP (BP) or saline are shown in (C). Significant differences in GR translocation rates are found between Stealth and BP, and between Stealth and Saline in OHCs (asterisks in C). Bars in (C) represent one standard deviation. 

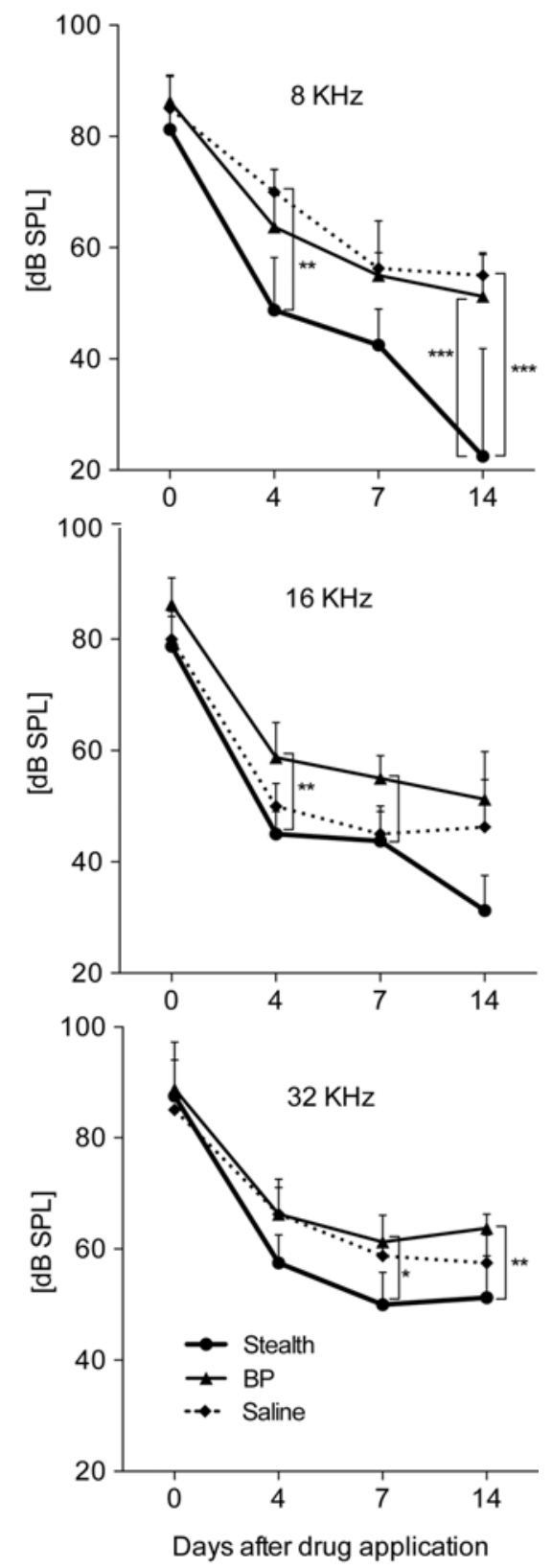

Fig. 4. ABR thresholds following saline, free BP or Stealth-Nano-BP.

Changes in ABR thresholds at 8,16 and $32 \mathrm{KHz}$ were determined on immediately after the treatment, 4, 7 and 14 days after treatment. Drug treatment showed significant effects on the ABR thresholds at each frequency by two-way ANOVA. Multiple comparisons with Bonferroni corrections identified significantly better ABR threshold in mice given Stealth-Nano-BP compared to mice given BP or saline at multiple time points shown by $*(p<0.05),{ }^{* *}(p<0.01)$ or $* * *(p<0.001)$. Bars represent one standard deviation. 

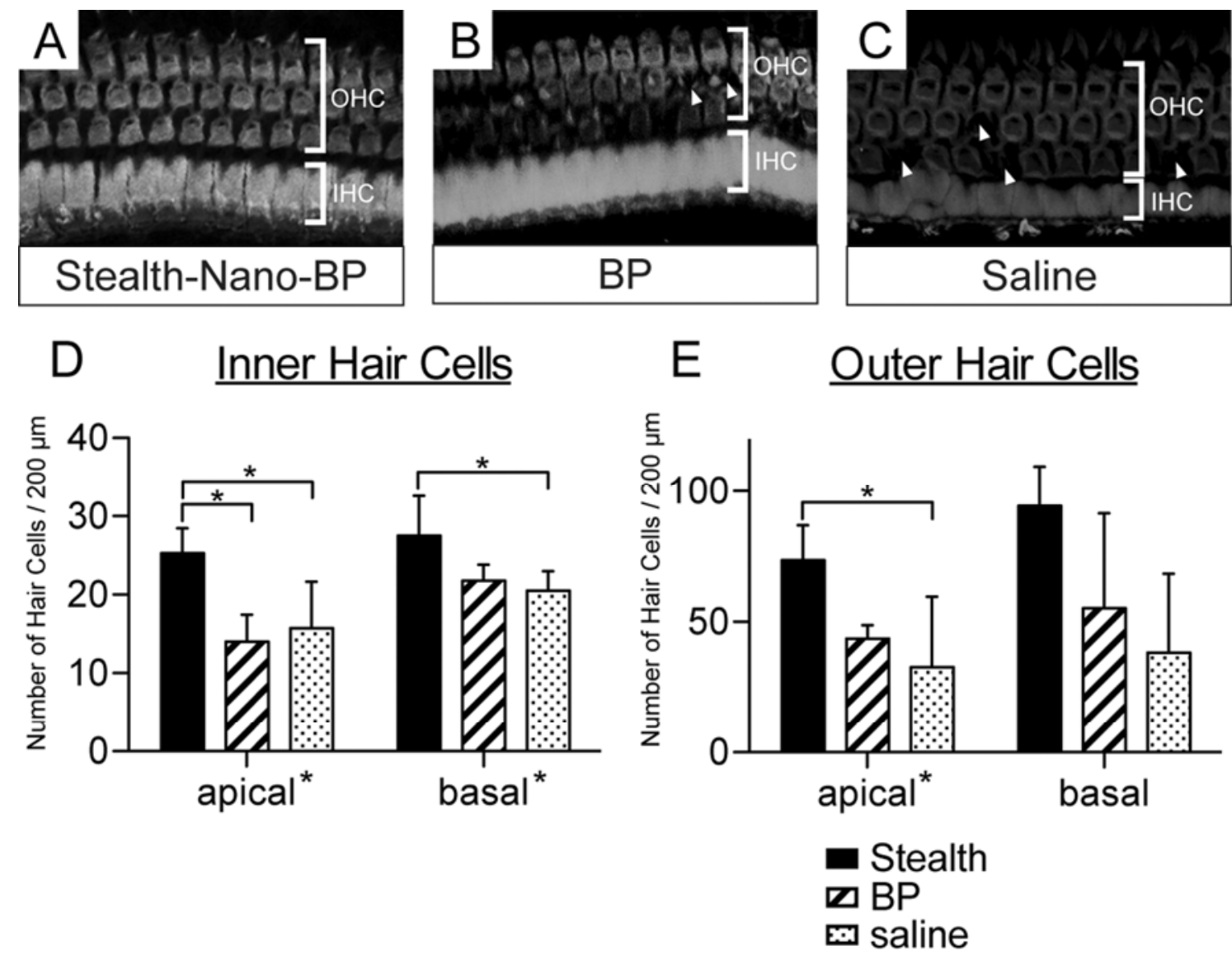

Fig. 5. Stealth-Nano-BP attenuates noise-induced damage on cochlear hair cells.

Phalloindin staining demonstrated well-maintained inner (IHC) and outer hair cells (OHC) in cochlear specimens of the basal portion treated with Stealth-Nano-BP (A), while in cochleae treated with free BP (B) or saline (C) exhibited the loss of OHCs (arrowheads). Numbers of remaining inner/outer hair cells in $200 \mu \mathrm{m}$ length in the apical and basal part of each cochlea were shown in D and E. Quantitative analyses showed that drug treatment had significant effects on the numbers of IHCs in the apical and the basal portions (D) and on the numbers of OHCs in the apical portion (E). Significant differences in the number of IHCs/OHCs by one-way ANOVA with Tukey's multiple comparison tests are indicated by asterisks. Bars represent one standard deviation. 


\section{Summary points}

- To examine the efficacy of stealth-nanoparticles encapsulating BP for the treatment of noise-induced sensorineural hearing loss (SNHL), pharmacokinetic, functional and histological analyses were performed using a mouse model of noise-induced SNHL.

- Histological analyses of rhodamine distribution demonstrated the efficacy of encapsulating a drug in stealth-nanoparticles for delivery to the cochlea.

- The pharmacokinetics of BP showed that encapsulating BP in stealth-nanoparticles, compared to administering free BP, is an effective strategy for sustained BP delivery to the cochlea.

- Immunohistochemistry for GR demonstrated a significant increase of GR nuclear translocation in OHCs of cochleae treated with stealth-nanoparticles encapsulating BP than those with free BP.

- Monitoring auditory function using ABRs demonstrated a significant therapeutic potential for stealth-nanoparticles encapsulating BP for noise-induced hearing loss.

- Functional analyses using ABRs showed that high doses of BP given as single injections had no therapeutic effects on the attenuation of noise-induced hearing loss.

- Histological analyses of cochlear hair cells showed that the systemic application of stealth-nanoparticles encapsulating BP significantly promoted hair cell survival after exposure to traumatic noise.

- A single injection of a high dose of free BP had no protective effects on cochlear hair cells exposed to loud noises.

- These results show that encapsulating BP in stealth-nanoparticles could greatly enhance the therapeutic efficacy of BP in acute SNHL. 